\title{
Radio emission in ultracool dwarfs: The nearby substellar triple system VHS 1256-1257
}

\author{
J. C. Guirado ${ }^{1,2}$, R. Azulay ${ }^{1,3}$, B. Gauza ${ }^{4,5,6}$, M. A. Pérez-Torres ${ }^{7,8}$, R. Rebolo ${ }^{4,5,9}$, \\ J. B. Climent ${ }^{1}$, and M. R. Zapatero Osorio ${ }^{10}$
}

\author{
${ }^{1}$ Departament d'Astronomia i Astrofísica, Universitat de València, C. Dr. Moliner 50, 46100 Burjassot, València, Spain \\ e-mail: guirado@uv.es \\ ${ }^{2}$ Observatori Astronòmic, Universitat de València, Parc Científic, C. Catedrático José Beltrán 2, 46980 Paterna, València, Spain \\ ${ }^{3}$ Max-Planck-Institut für Radioastronomie, Auf dem Hügel 69, 53121 Bonn, Germany \\ ${ }^{4}$ Instituto de Astrofísica de Canarias, 38200 La Laguna, Tenerife, Spain \\ 5 Departamento de Astrofísica, Universidad de La Laguna, 38206 La Laguna, Tenerife, Spain \\ ${ }^{6}$ Departamento de Astronomía, Universidad de Chile, Camino el Observatorio 1515, Casilla 36-D, Las Condes, Santiago, Chile \\ ${ }^{7}$ Instituto de Astrofísica de Andalucía (IAA, CSIC), Glorieta de la Astronomía, s/n, 18008 Granada, Spain \\ ${ }^{8}$ Departamento de Física Teórica, Facultad de Ciencias, Universidad de Zaragoza, 50009 Zaragoza, Spain \\ ${ }^{9}$ Consejo Superior de Investigaciones Científicas, 28006 Madrid, Spain \\ ${ }^{10}$ Centro de Astrobiología (CSIC-INTA), Crta. Ajalvir km 4, 28850 Torrejón de Ardoz, Madrid, Spain
}

Received 19 October 2017 / Accepted 1 December 2017

\begin{abstract}
Aims. With the purpose of investigating the radio emission of new ultracool objects, we carried out a targeted search in the recently discovered system VHS J125601.92-125723.9 (hereafter VHS 1256-1257); this system is composed by an equal-mass M7.5 binary and a L7 low-mass substellar object located at only $15.8 \mathrm{pc}$.

Methods. We observed in phase-reference mode the system VHS 1256-1257 with the Karl G. Jansky Very Large Array at X band and $L$ band and with the European VLBI Network at $L$ band in several epochs during 2015 and 2016.

Results. We discovered radio emission at $X$ band spatially coincident with the equal-mass M7.5 binary with a flux density of $60 \mu \mathrm{Jy}$. We determined a spectral index $\alpha=-1.1 \pm 0.3$ between 8 and $12 \mathrm{GHz}$, suggesting that non-thermal, optically thin, synchrotron, or gyrosynchrotron radiation is responsible for the observed radio emission. Interestingly, no signal is seen at $L$ band where we set a $3 \sigma$ upper limit of $20 \mu \mathrm{Jy}$. This might be explained by strong variability of the binary or self-absorption at this frequency. By adopting the latter scenario and gyrosynchrotron radiation, we constrain the turnover frequency to be in the interval $5-8.5 \mathrm{GHz}$, from which we infer the presence of kG-intense magnetic fields in the M7.5 binary. Our data impose a $3 \sigma$ upper bound to the radio flux density of the L7 object of $9 \mu \mathrm{Jy}$ at $10 \mathrm{GHz}$.
\end{abstract}

Key words. brown dwarfs - stars: magnetic field - radiation mechanisms: general - techniques: interferometric

\section{Introduction}

Radio observations play an important role to understand the processes involved in the formation and evolution of stellar and substellar objects. In particular, radio emission studies of ultracool objects (late M, L, and T objects; e.g., Matthews 2013; Kao et al. 2016, and references therein) are relevant to probe the magnetic activity of these objects and its influence on the formation of disks or planets. Moreover, the study of ultracool dwarfs may open a suitable route to the detection of radio emission of exoplanets. While no exoplanet has been yet detected at radio wavelengths, an increasing number of ultracool objects (McLean et al. 2012) show substantial evidence of radio emission at $\mathrm{GHz}$ frequencies in objects with spectral types as cool as T6.5 (Kao et al. 2016). But radio detection of ultracool dwarfs is still a relatively rare phenomenon, and new candidates are needed to improve the statistics of active cool objects. One of these targets is the system VHS J125601.92125723.9 (hereafter VHS 1256-1257; Gauza et al. 2015); this system is composed by a $0.1^{\prime \prime}$ equal-magnitude M7.5 binary (components A and B; Stone et al. 2016) and a lower mass L7 companion (component b) separated 8 " from the primary pair. The system is relatively young, 150-300 Myr, and nearby (12.7-17.1 pc); this locates the low-mass object $b$ near the deuterium burning limit (Stone et al. 2016). Recently, Zapatero Osorio et al. (2017, in prep.) have determined a new trigonometric distance of $15.8_{-0.8}^{+1.0} \mathrm{pc}$ for the system using optical and near-infrared images spanning a few years. This distance is compatible with a likely age of $300 \mathrm{Myr}$, which agrees with the strong lithium depletion observed in the high resolution spectra of the M7.5 binary and the recent age determinations of Stone et al. (2016) and Rich et al. (2016). In this work, we adopt these values of distance and age. Using the bolometric corrections available for M7-M8 and red L dwarfs (Golimowski et al. 2004; Filippazzo et al. 2015) and the new distance, the luminosities are determined at $\log L / L_{\odot}=$ $-4.91 \pm 0.10$ dex for the faint L7 companion VHS $1256-1257 \mathrm{~b}$ and $\log L / L_{\odot}=-3.24 \pm 0.10 \mathrm{dex}$ for each member of the M7.5 binary. By applying the luminosity - mass - age relationship of Chabrier et al. (2000), we infer masses in the interval 10-20 $M_{\text {Jup }}$ (VHS 1256-1257 b) and 50-80 $M_{\text {Jup }}$ (A and B components) for the age range $150-300 \mathrm{Myr}$ with most likely values of $15-20 M_{\text {Jup }}$ and $70-80 M_{\text {Jup }}$ at $300 \mathrm{Myr}$. The interest in VHS 1256-1257 is obvious for several reasons: first, it is only 
Table 1. VLA and VLBI observations of VHS 1256-1257.

\begin{tabular}{|c|c|c|c|c|c|c|c|}
\hline Telescope/configuration & Epoch & Frequency band & UT range & Beam size & $\begin{array}{l}\mathrm{PA} \\
{\left[{ }^{\circ}\right]}\end{array}$ & $\begin{array}{c}\mathrm{rms} \\
{[\mu \mathrm{Jy}]}\end{array}$ & $\begin{array}{l}\text { Peak } \\
{[\mu \mathrm{Jy}]}\end{array}$ \\
\hline VLA/BnA & 15 May 2015 & $X$ & $05: 10-06: 10$ & $0.78^{\prime \prime} \times 0.45^{\prime \prime}$ & -51 & 3 & 60 \\
\hline VLA/B & 28 Jul 2016 & $L$ & 00:00-01:00 & $5.66^{\prime \prime} \times 3.38^{\prime \prime}$ & -12 & 7 & $<21$ \\
\hline $\mathrm{EVN}^{a}$ & 4 Mar 2016 & $L$ & $22: 30-04: 30$ & $3.1 \times 2.4 \mathrm{mas}$ & -64 & 22 & $<66$ \\
\hline " & 27 May 2016 & $"$ & $15: 30-23: 30$ & $11.9 \times 2.2 \mathrm{mas}$ & -78 & 30 & $<90$ \\
\hline$"$ & 2 Nov 2016 & $"$ & $05: 00-13: 00$ & $3.2 \times 2.3 \mathrm{mas}$ & -73 & 31 & $<93$ \\
\hline
\end{tabular}

Notes. ${ }^{(a)}$ European VLBI Network using the following antennas: Jodrell Bank, Westerbork, Effelsberg, Medicina, Noto, Onsala85, Tianma65, Urumqi, Torun, Zelenchukskaya, Hartebeesthoek, Sardinia, Irbene, and DSS63.

the third multiple system in which all three components may be substellar (Bouy et al. 2005; Radigan et al. 2013) second, the L7 source belongs to one intriguing (not yet understood) population of very red L dwarfs with likely high content of atmospheric dust or high metallicity (Rich et al. 2016); third, given their large separation $\left(8^{\prime \prime}\right)$, unambiguous observations of the substellar object $b$ and the central pair $\mathrm{AB}$ are accessible by instruments at virtually all wavelengths, including radio; and fourth, the binarity of the host system $\mathrm{AB}$ permits the determination of their dynamical masses in a few years, which is essential to fully characterize the system. Additionally, Gauza et al. (2015) reports detection of the $\mathrm{H}_{\alpha}$ line emission $(656.3 \mathrm{~nm})$ in the primary, which indicates the existence of chromospheric activity in this M7.5 low-mass binary, therefore showing the ability to sustain significant magnetic fields, and hence, radio emission.

In this paper we present Karl G. Jansky Very Large Array (VLA) and European very-long-baseline interferometry (VLBI) Network (EVN) observations of VHS 1256-1257. We describe our observations and report the principal results, consisting in the discovery of the radio emission of the central components of the VHS 1256-1257 system. We also present a study of the spectral behavior of the detected emission and set an upper bound to the possible radio emission of the very low-mass companion VHS 1256-1257 b.

\section{Observations and data reduction}

\subsection{VLA observations}

We observed with DDT/Exploratory Time with the VLA the system VHS $1256-1257$ at $X$ band and $L$ band on 2015 May 15 and 2016 July 28, respectively. The observation at $X$ band lasted $2 \mathrm{~h}$ and was carried out in $\mathrm{BnA}$ configuration, using an effective bandwidth of $4 \mathrm{GHz}(8-12 \mathrm{GHz})$ in dual polarization. The observation at $L$ band lasted $1 \mathrm{~h}$, in B configuration, and using an effective bandwidth of $1 \mathrm{GHz}(1-2 \mathrm{GHz})$ in dual polarization (see Table 1). We used 3C286 as absolute flux calibrator meanwhile we performed amplitude and phase calibration using interleaved observations of the radio source J1254-1317. Data reduction and imaging were carried out using the CASA ${ }^{1}$ software package of the NRAO. The standard procedure of calibration for continuum VLA data was applied. Special care was taken to flag data contaminated by radio frequency interferences (RFI) at $L$ band. The resulting images are shown in Fig. 1.

\subsection{VLBI observations}

The VLA observations explained above confirmed the radio emission of VHS 1256-1257. This detection triggered VLBI

\footnotetext{
1 http://casa.nrao.edu
}

observations that were carried out with the EVN at $L$ band (1.6 GHz; see Table 1) with the purpose of constraining both the origin and properties of the radio emission. Each observation lasted $6-7 \mathrm{~h}$ and both polarizations were recorded with a rate of $1024 \mathrm{Mbps}$ (two polarizations, eight subbands per polarization, $16 \mathrm{MHz}$ per subband, two bits per sample). We used the phasereference mode and the selected calibrators were J1254-1317 (as primary calibrator, separated $0.5^{\circ}$ from VHS $1256-1257$ ), and J1303-1051 (as secondary calibrator, separated $2.7^{\circ}$ ). The duty cycle was $1 \mathrm{~min}$ on the primary calibrator, $3 \mathrm{~min}$ on the target, and 1 min on the secondary calibrator with a total integration time at the target of $\sim 3.5 \mathrm{~h}$ per epoch.

The data reduction was realized using the program Astronomical Image Processing System (AIPS) of the National Radio Astronomy Observatory (NRAO) with standard routines. Once the final data were obtained, the images were made with the Caltech imaging program DIFMAP (Shepherd et al. 1994). We did not detect either the central M7.5 binary or the very low-mass substellar companion at any epoch, establishing an average flux density upper limit of $\sim 80 \mu \mathrm{Jy}(3 \sigma)$. The interpretation of these non-detections are discussed in next section.

\section{Results and discussion}

\subsection{Radio emission of the central pair VHS 1256-1257 AB}

Figure 1 revealed a clear detection on 2015 May 15 ( $X$-band) of an unresolved source with a peak flux density of $60 \mu \mathrm{Jy}$, which can be assigned to the primary of VHS 1256-1257, the equalmass M7.5 binary. We confirmed this identification using both the coordinates and proper motion given in Gauza et al. (2015) to find the expected position of component $\mathrm{AB}$ at the time of our observation; this expected position differs only $0.18^{\prime \prime}$, about onethird of the synthesized beam, from the measured position in the VLA $X$-band map in Fig. 1 (the source has moved $\sim 6.3^{\prime \prime}$ between Gauza's epoch and ours). A noise floor of $\sim 3 \mu \mathrm{Jy}$ imposes a strong upper bound to the radio emission at the expected position of the low-mass companion VHS 1256-1257 b. In contrast, we found no detection in any of the components of the system on 2016 July 28 ( $L$ band) with a $3 \sigma$ threshold detection of $20 \mu \mathrm{Jy}$. The flux density measured at $X$ band implies a radio luminosity of $1.95 \times 10^{-13} \mathrm{erg} \mathrm{s}^{-1} \mathrm{~Hz}^{-1}$ at $15.8 \mathrm{pc}$. Assuming that the flux is originated at only one of the central components of VHS 1256-1257, this luminosity is similar to other single ultracool dwarfs detected with comparable spectral types (M7.5; McLean et al. 2012). We notice that the figures above are halved if we consider both components to contribute equally to the radio flux. We did not detect significant traces of variability or pulsed emission in the flux density throughout the $2 \mathrm{~h}$ duration of our observations, which suggests that the detected radio 

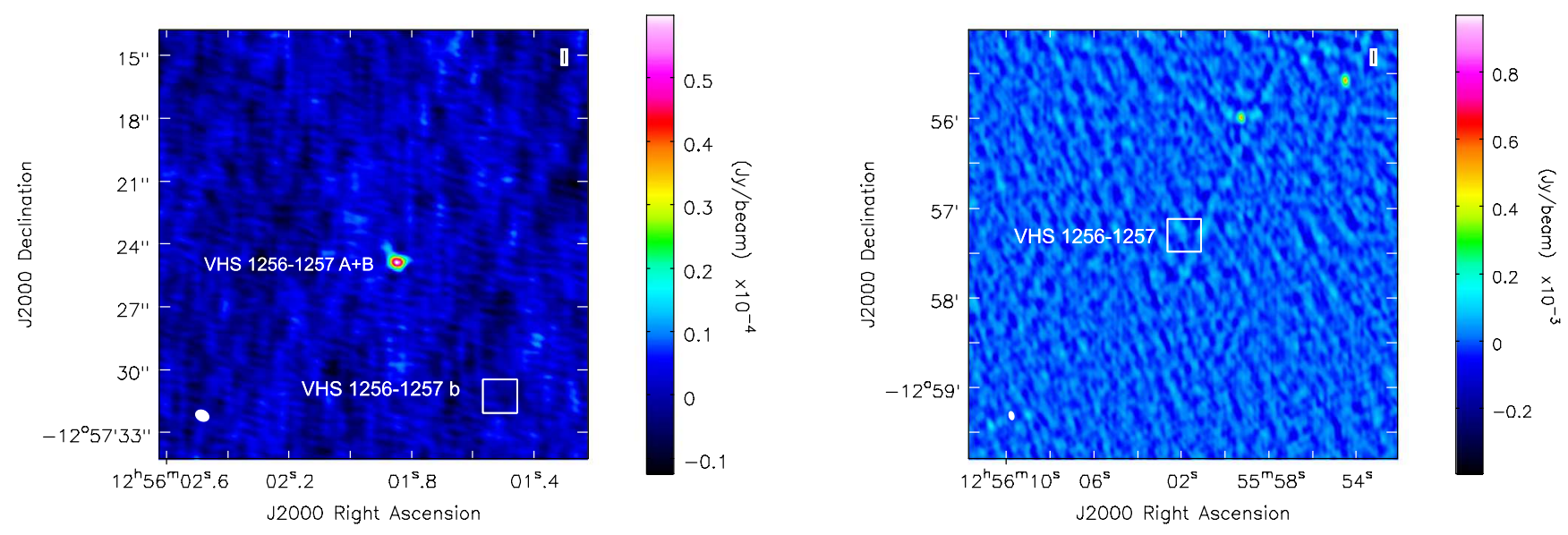

Fig. 1. Left: VLA image of the VHS 1256-1257 field at $X$-band. The detected source is readily assigned to the M7.5 binary VHS $1256-1257$ AB. The location of the (undetected) L7-object b is marked with a solid white box. The $3 \sigma$ threshold detection is $9 \mu \mathrm{Jy}$. The restoring beam, shown at the bottom left corner, is an elliptical Gaussian of $0.78 \times 0.45 \operatorname{arcsec}\left(\mathrm{PA}-51^{\circ}\right)$. At $15.8 \mathrm{pc}$, the separation between components $\mathrm{AB}$ and $\mathrm{b}$ corresponds to 128.4 AU. Right: VLA image of the VHS 1256-1257 field at $L$-band. A solid box, with size that of the $X$-band image, is centered at the position of the $X$-band detection. None of the VHS 1256-1257 components is detected at this frequency band. The $3 \sigma$ threshold detection is $20 \mu \mathrm{Jy}$. The two bright knots seen in the map at the NW correspond to known extragalactic radio sources. The restoring beam, shown at the bottom left corner, is an elliptical Gaussian of $5.66 \times 3.38 \operatorname{arcsec}\left(\mathrm{PA}-12^{\circ}\right)$.

emission is produced either in quiescent conditions or, alternatively, during a long-duration, energetic flare. However, the latter possibility seems unlikely given the low frequency rate of energetic flares in late $\mathrm{M}$ dwarfs $(\sim 0.1 /$ day) recently reported by Gizis et al. (2017).

Obtaining an estimate of the brightness temperature is difficult since the resolution of our observations does not provide a precise estimate of the size of the emitting region. Additionally, as said above, the fraction of the radio emission that is originated at each component of the central binary is unknown. Under the assumption that radio emission comes from a single object of size $0.12 R_{\odot}$ (derived from the models of Chabrier et al. 2000), we calculate a brightness temperature of $5.4 \times 10^{7} \mathrm{~K}(\times 1 / 2$ for equal binary contribution) that is consistent with synchrotron or gyrosynchrotron non-thermal radio emission (Dulk 1985). In principle, the low degree of circular polarization (less than $15 \%$ ) seems to discard coherent mechanisms predicted for ultracool dwarfs (i.e., auroral emissions; Hallinan et al. 2015; Kao et al. 2016), which are normally associated with a high degree of polarization; however, in the case in which both components A and $\mathrm{B}$ contribute to the radio emission, we notice that the degree of circular polarization above would be the result of the combination of both radio emitters, not properly reflecting the polarization properties of each one. Further information about the emission mechanism acting on this object can be obtained from the $4 \mathrm{GHz}$ recorded bandwidth of our $X$-band VLA observations. In practice, we produced four narrower band images of VHS 1256-1257 by deconvolving adjacent $1 \mathrm{GHz}$ bandwidth data sets separately (see Fig. 2), from which we could obtain an indication of the spectral behavior of this system between 8 and $12 \mathrm{GHz}$. The corresponding spectral index is $\alpha=-1.1 \pm 0.3\left(S \propto v^{\alpha}\right)$; this is compatible with optically thin non-thermal synchrotron or gyrosynchrotron emission from a power-law energy distribution of electrons, which indicates, in turn, that strong magnetic fields play an active role in this system.

If the optically thin regime were held down to frequencies as low as $1.4 \mathrm{GHz}$, we should have detected radio emission in VHS 1256-1257 with a flux density above $300 \mu \mathrm{Jy}$ (actually, this was the motivation for the $1.6 \mathrm{GHz}$ VLBI observations reported in Sect. 2.2); however, no flux above $20 \mu \mathrm{Jy}$ is detected at the nominal position of VHS 1256-1257 at $L$ band. Ultracool dwarfs have shown to be strongly variable source in radio (Bower et al. 2016), therefore arguments of variability could explain this lack of detection. However, the persistent nondetection in our VLBI observations (with a noise floor 10 times smaller than the expected $1.6 \mathrm{GHz}$ flux density according to the spectral index derived) led us to formulate a different hypothesis consisting in considering that the radio emission is actually self-absorbed at the frequency of $1.4 \mathrm{GHz}$.

We have further explored this hypothesis following the analytic expressions developed by White et al. (1989; W89) for gyrosynchrotron radio emission of dMe stars in quiescent conditions, which provide estimates of the spectral index for the optically thick and thin components, and, therefore, the turnover frequency. We notice that synchrotron radio emission is not ruled out by our data, but gyrosynchrotron from midly relativistic electrons seems to be the preferred mechanism for previously studied M-dwarfs (i.e., Osten \& Jayawardhana 2006; Osten et al. 2009), which in turn justifies the use of W89 formulation. These authors assume a dipolar magnetic field, which scales as $B(r) \propto r^{-n}$, where $n=3$ and $r$ are the distance measured from the dipole and a power-law electron distribution $N^{\prime}(E)$, which also scales as $N(E) \propto N_{o}^{\prime}(E) r^{-m}$, where the index $m$ varies from 0 (isotropic electron distribution) to $m=3$ ( $=n$, the radial dependence of the electron distribution is the same as that of the magnetic field). Taking our measured optically thin spectral index $(\alpha=-1.1 \pm$ 0.3 ), which implies an energy index $\delta=2.6$, W89 expressions provide two values for the spectral index of the optically thick component of the radiation, $\alpha=0.6$, and $\alpha=1.2$, corresponding to the two extreme cases of $m=0$, and $m=3$, respectively. With the constraints above, we can set lower bounds to the turnover frequency of $\sim 8 \mathrm{GHz}(m=0)$ and $\sim 5 \mathrm{GHz}(m=3)$, effectively limiting the turnover frequency to the range $5-8.5 \mathrm{GHz}$ (Fig. 3).

In addition, for gyrosynchrotron emission, the turnover frequency depends strongly on the magnetic field (and very weakly of the rest of the model parameters, $m$ and $n$ in particular; W89) in the form $B \sim 150 v^{1.3}$, which provides magnetic field intensities for the previous turnover frequency 


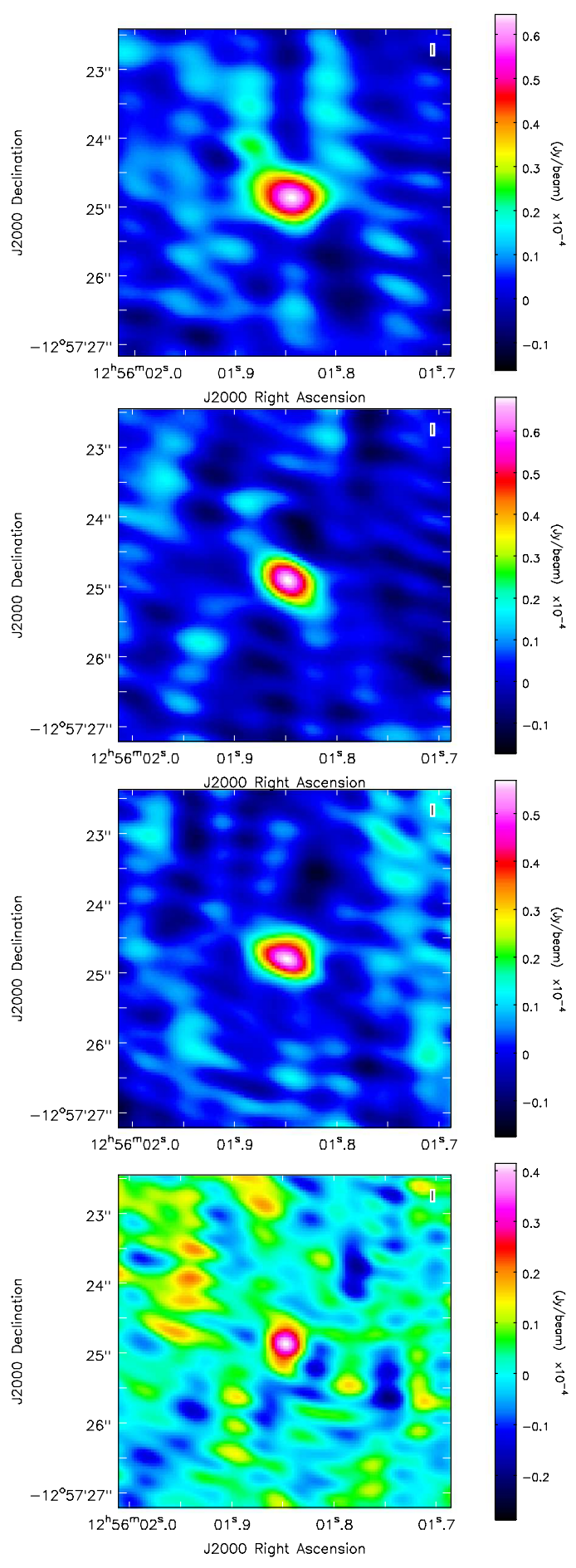

Fig. 2. VLA images the VHS 1256-1257 system (unresolved central binary $\mathrm{AB}$ ) at $X$ band made from $1 \mathrm{GHz}$ subsets of the total data. From top to bottom, the center frequencies are $8.5,9.5,10.5$, and $11.5 \mathrm{GHz}$, respectively. The peak flux density decreases from $63 \mu \mathrm{Jy}$ (top) to $45 \mu \mathrm{Jy}$ (bottom image).

range of $1.2-2.2 \mathrm{kG}$. Interestingly, the previous values agree with M-dwarf magnetic field estimates derived from theoretical models (Reiners \& Christensen 2010): from the luminosity and mass reported for the components of VHS 1256-1257A and B (Stone et al. 2016; Rich et al. 2016), and using the radius of $0.12 R_{\odot}$ the model of Reiners \& Christensen (2010) provides values of the dipole field of $\sim 1.7 \mathrm{kG}$, which is well within the range derived previously. This estimate is near the average value of

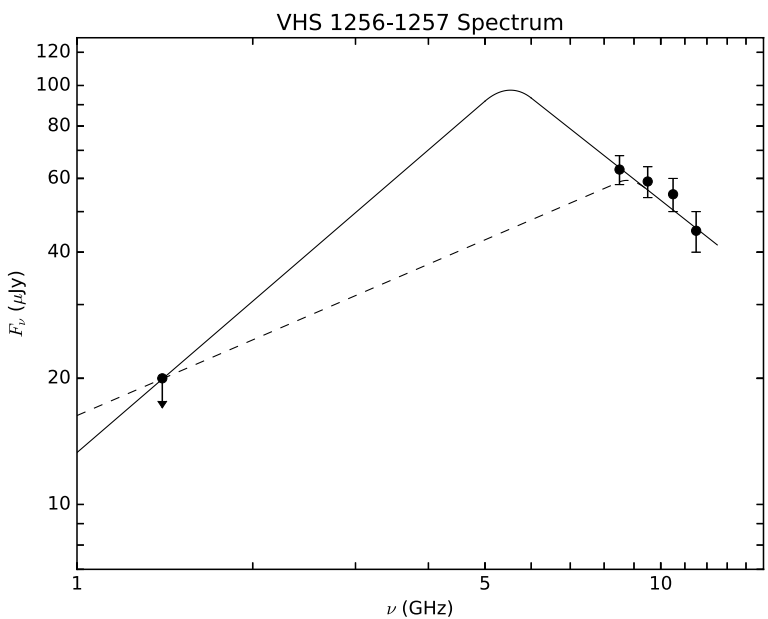

Fig. 3. Spectrum of VHS 1256-1257 from VLA observations. The flux densities between 8 and $12 \mathrm{GHz}$ correspond to the maps shown in Fig. 2 . The upper bound resulting from the non-detection at $L$ band is denoted with a downward arrow. The solid lines illustrate two possible spectra, each one made from a combination of the behavior predicted by the White et al. (1989) model of gyrosynchrotron emission (optically thick regime, showing the two extreme cases of $\alpha=0.6$ : dashed line and 1.2: solid line), and a fit to our flux density measurements (optically thin regime, $\alpha=-1.1$ ).

the magnetic field intensity found in a sample of M7-9.5 dwarfs (Reiners \& Basri 2010).

\subsection{Spectral energy distribution of VHS 1256-1257 AB}

Figure 4 shows the complete spectral energy distribution (SED) of VHS 1256-1257AB covering from VLA through optical observations. The data at visible, near- and mid-infrared wavelengths are taken from Gauza et al. (2015). The observed spectrum is conveniently flux calibrated using the 2MASS $J H K$ magnitudes and the zero points given in Cohen et al. (2003). The W4 photometry reported in Gauza et al. (2015) is affected by a large uncertainty indicative of $S / N \leq 4$. Therefore, we adopt the nominal sensitivity limit of the WISE mission at $22 \mu \mathrm{m}$ (Wright et al. 2010). The BT-Settl solar metallicity model atmosphere (Baraffe et al. 2015) computed for $T_{\text {eff }}=2600 \mathrm{~K}$ and $\log g=5.0\left[\mathrm{~cm} \mathrm{~s}^{-2}\right]$ is also included in Fig. 4 to illustrate the expected photospheric emission at frequencies not covered by the observations. The synthetical spectrum is normalized to the $J$-band emission of VHS 1256-1257AB. This temperature and surface gravity are expected for dwarfs near the star-brown dwarf borderline with an age of a few hundred Myr (Chabrier et al. 2000). They also agree with the spectral type $T_{\text {eff }}$ relationship defined for high-gravity, ultracool dwarfs by Filippazzo et al. (2015) and Faherty et al. (2016). The BT-Settl photospheric model extends from $\sim 300$ up to $\sim 7.5 \times 10^{5} \mathrm{GHz}$ and does not overlap in the frequency axis with the VLA observations. A linear extrapolation of the theoretical SED down to $10 \mathrm{GHz}$ yields a predicted photospheric flux of $\sim 8.3 \times 10^{-7} \mathrm{mJy}$. The observed VLA $X$-band flux is $\sim 65800$ times higher than the expected photospheric emission suggesting that the mechanism responsible for the emission at $10 \mathrm{GHz}$ is extremely powerful.

\subsection{Radio emission of the very low-mass companion VHS 1256-1257b}

Our non-detection at $X$ band put a strong upper bound to the flux density of this L7 object of $9 \mu \mathrm{Jy} \quad(3 \sigma)$. 


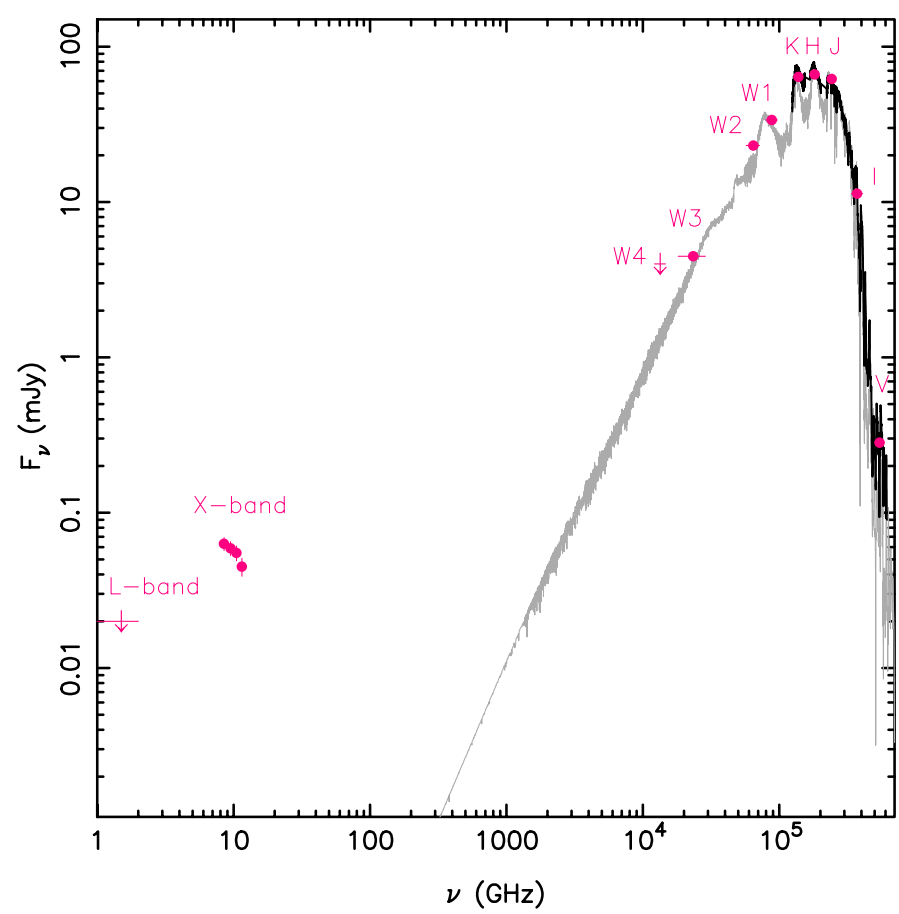

Fig. 4. Observed spectral energy distribution of the unresolved binary VHS 1256-1257AB from optical wavelengths through $1 \mathrm{GHz}$. The optical and near-infrared observed spectra (Gauza et al. 2015) are shown with a solid black line, while the photometric observations are plotted as pink symbols: solid circles stand for actual detections with $S / N \geq 4$ and arrows represent $4 \sigma$ upper limits. The filter and passband names are labeled. The horizontal error bars represent the width of the filters. Also plotted is the BT-Settl photospheric model (gray line; Baraffe et al. 2015) normalized to the $J$-band flux of VHS 1256-1257AB. This model corresponds to a cool dwarf with solar metallicity, $T_{\text {eff }}=2600 \mathrm{~K}$, and $\log g=5.0\left[\mathrm{~cm} \mathrm{~s}^{-2}\right]$, which are the parameters expected for an M7.5 source $\left(0.06-0.072 M_{\odot}\right)$ with an age of a few hundred Myr (Chabrier et al. 2000).

Certainly, the ultracool dwarf samples carried out by McLean et al. (2012) and Route \& Wolszczan (2013) show that radio detections at $\mathrm{GHz}$ frequencies are not frequent for objects later than L3.5, which could be expected by the declining activity of the cooler atmospheres of these objects. Despite this, auroral radio emission (based on electron cyclotron maser emission mechanism) has been detected in a number of late $\mathrm{L}$ and $\mathrm{T}$ dwarfs (i.e., Hallinan et al. 2015; Kao et al. 2016; Pineda et al. 2017). Could this emission be expected in VHS 1256-1257 b? At a distance of $15.8 \mathrm{pc}$, the reported luminosities of the coolest dwarfs detected in radio (Pineda et al. 2017) would produce a quiescent emission of $\sim 4 \mu \mathrm{Jy}$ (below our $3 \sigma$ detection level). In case of auroral, pulsed emission, the radio flux would significantly rise up to peaks of $100 \mu \mathrm{Jy}$, a factor of $\sim 10$ above our detection limit in $X$ band. However, we do not see such a peak in our data indicating that, at the moment of the VLA observations, VHS 1256-1257 b did not show strong levels of auroral activity similar to those seen in other ultracool dwarfs. Kao et al. (2016) found a strong correlation between radio aurorae and the presence of the $\mathrm{H}_{\alpha}$ line; however, only $7-13 \%$ of the dwarfs with spectral types between L4 and T8 display $\mathrm{H}_{\alpha}$ emission (Pineda et al. 2016). Given the absence of $\mathrm{H}_{\alpha}$ in VHS 1256-1257 b, the statistics above do not favor the presence of auroral radio emission in this object.

On the other hand, considering the $\sim \mathrm{kG}$ estimate of the magnetic field for VHS 1256-1257AB, along with the fact that this triple system could have formed from collapse and fragmentation of the same rotating cloud, component $\mathrm{b}$ may have also retained high levels of rotation and magnetic field, which eventually may produce sustainable radio emission, although variable, explaining our non-detection. Indeed, both the model of Reiners \& Christensen (2010) and the scaling law reported in Christensen et al. (2009) (magnetic field $\propto$ energy flux, valid for fully convective, rapidly rotating objects) predict magnetic fields $>10^{2} \mathrm{G}$ for an object with mass as low as 10-20 $M_{\text {Jup }}$, and effective temperature of $800-1000 \mathrm{~K}$ (Gauza et al. 2015; Rich et al. 2016).

Additionally, we can estimate the possible radio emission of VHS 1256-1257 b from the Nichols et al. (2012) model. These authors consider the auroral emission as originating from magnetosphere-ionosphere coupling currents resulting from an angular velocity shear in a fast-rotating magnetized object. By assuming the fiducial parameters given in Nichols et al. (2012) (corresponding to a Jovian-like plasma), a magnetic field of $\sim 2 \mathrm{kG}$, and a rotation period of $\sim 2 \mathrm{~h}$ (as extracted from the distribution of brown dwarf rotational periods given in Metchev et al. 2015), we find that VHS 1256-157 b may present auroral emission with a peak flux density of $\sim 100 \mu \mathrm{Jy}$, coincident with the estimate above resulting from the Pineda et al. (2017) compilation. However, since the currents proposed by Nichols et al. (2012) are created through magnetic field reconnections, the cool atmosphere of VHS 1256-157 b may hamper the existence of auroral emission, as there is evidence that magnetic reconnections are not allowed or are suppressed at temperatures below $\sim 1500 \mathrm{~K}$ (Gizis et al. 2017).

\section{Conclusions}

We have reported the detection of radio emission from the VHS 1256-1257 system. Given the youth of the system $(\sim 300 \mathrm{Myr})$, its proximity, $(15.8 \mathrm{pc})$, architecture (a possible triple substellar system), and presence of a very low-mass substellar object at $8^{\prime \prime}$ from the primary, this detection appears relevant to study the role of the magnetic field in brown dwarfs. The radio emission is originated at the central system $A B$, likely consisting in non-thermal synchrotron or gyrosynchrotron emission in the presence of kG-intense magnetic field. Further monitoring of the system at intermediate frequencies to those presented here should confirm our finding that the turnover frequency of the radiation is located between 5 and $8.5 \mathrm{GHz}$. The use of interferometers with higher resolution (eMERLIN or EVN at $5-8 \mathrm{GHz}$ ) should discriminate if the radio emission originates in one of the components $(\mathrm{A}$ or $\mathrm{B})$, in both $(\mathrm{A}+\mathrm{B})$, or perhaps a sort of interaction between them. These higher resolution studies will open the door to a multiepoch astrometric study directed to the determination of the parallax of the system (modest 5 mas precise positions would result in a $1 \mathrm{pc}$ precise distance) and, additionally, to precise estimates of the masses of the internal pair via monitoring of its orbital motion $(4.5 \mathrm{yr}$ period for a faceon orbit). VHS $1256-1257 \mathrm{~b}$ is not seen in our maps; however, despite our non-detection at the level of $9 \mu \mathrm{Jy}, \sim 100 \mathrm{G}$ magnetic fields are expected in this 10-20 $M_{\text {Jup }}$ object. Therefore, the presence of GHz-radio emission VHS 1256-1257 b should be further explored as this would provide useful constraints to the emission mechanism in the coolest substellar objects.

Acknowledgements. We thank Iván Martí-Vidal and Eskil Varenius (Chalmers University of Technology) for helping with the $L$-band VLA data reduction. The National Radio Astronomy Observatory is a facility of the National Science Foundation operated under cooperative agreement by Associated Universities, 
Inc. The European VLBI Network is a joint facility of independent European, African, Asian, and North American radio astronomy institutes. Scientific results from data presented in this publication are derived from the EVN project code EG092. J.C.G., R.A., and J.B.C. were partially supported by the Spanish MINECO projects AYA2012-38491-C02-01 and AYA2015-63939-C2-2-P and by the Generalitat Valenciana projects PROMETEO/2009/104 and PROMETEOII/2014/057. B.G. acknowledges support from the CONICYT through FONDECYT Fellowship grant No. 3170513. M.A.P.T. acknowledges support from the Spanish MINECO through grant AYA2015-63939-C2-1-P.

\section{References}

Baraffe, I., Homeier, D., Allard, F., \& Chabrier, G., 2015, A\&A, 577, A42 Bouy, H., Martín, E. L., Brandner, W., \& Bouvier, J., 2005, AJ, 129, 511 Bower, G. C., Loinard, L., Dzib, S., et al. 2016, ApJ, 830, 107

Chabrier, G., Baraffe, I., Allard, F., \& Hauschildt, P. 2000, ApJ, 542, 464

Christensen, U. R., Holzwarth, V., \& Reiners, A. 2009, Nature, 457, 167

Cohen, M., Wheaton, W. A., \& Megeath, S. T. 2003, AJ, 126, 1090

Dulk, G. A. 1985, ARA\&A, 23, 169

Faherty, J. K., Riedel, A. R., Cruz, K. L., et al. 2016, ApJS, 225, 10

Filippazzo, J. C., Rice, E. L., Faherty, J., et al. 2015, ApJ, 810, 158
Gauza, B., Béjar, V. J. S., Pérez-Garrido, A., et al. 2015, ApJ, 804, 96 Gizis, J. E., Paudel, R. R., Mullan, D., et al. 2017, ApJ, 845, 33

Golimowski, D. A., Leggett, S. K., Marley, M. S., et al. 2004, AJ, 127, 3516 Hallinan, G., Littlefair, S. P., Cotter, G., et al. 2015, Nature, 523, 568

Kao, M. M., Hallinan, G., Pineda, J. S., et al. 2016, ApJ, 818, 24 Matthews, L. D. 2013, PASP, 125, 313

McLean, M., Berger, E., \& Reiners, A. 2012, ApJ, 746, 23

Metchev, S. A., Heinze, A., Apai, D., et al. 2015, ApJ, 799, 154

Nichols, J. D., Burleigh, M. R., Casewell, S. L., et al. 2012, ApJ, 760, 59

Osten, R. A., \& Jayawardhana, R. 2006, ApJ, 644, L67

Osten, R. A., Phan-Bao, N., Hawley, S. L., Reid, I. N., \& Ojha, R. 2009, ApJ, 700,1750

Pineda, J. S., Hallinan, G., Kirkpatrick, J. D., et al. 2016, ApJ, 826, 73

Pineda, J. S., Hallinan, G., \& Kao, M. M. 2017, ApJ, 846, 75

Radigan, J., Jayawardhana, R., Lafrenière, D., et al. 2013, ApJ, 778, 36

Reiners, A., \& Basri, G. 2010, ApJ, 710, 924

Reiners, A., \& Christensen, U. R. 2010, A\&A, 522, A13

Rich, E. A., Currie, T., Wisniewski, J. P., et al. 2016, ApJ, 830, 114

Route, M., \& Wolszczan, A. 2013, ApJ, 773, 18

Shepherd, M. C., Pearson, T. J., \& Taylor, G. B. 1994, BAAS, 26, 987

Stone, J. M., Skemer, A. J., Kratter, K. M., et al. 2016, ApJ, 818, L12

White, S. M., Kundu, M. R., \& Jackson, P. D. 1989, A\&A, 225, 112

Wright, E. L., Eisenhardt, P. R. M., Mainzer, A. K., et al. 2010, AJ, 140, 1868 